\title{
Double-Stranded RNA Binding Domain
}

National Cancer Institute

\section{Source}

National Cancer Institute. Double-Stranded RNA Binding Domain. NCI Thesaurus. Code C25958.

The Double-Stranded RNA Binding Domain is found in a variety of RNA-binding proteins with different structures and exhibiting a diversity of functions. It is involved in localization of at least five different mRNAs in the early Drosophila embryo and by interferon-induced protein kinase in humans, which is part of the cellular response to dsRNA. (InterPro IPR001159) 